\title{
Treatment of Intermediate-Stage Hepatocellular Carcinoma in Japan: Position of Curative Therapies
}

\author{
Kazuya Kariyama ${ }^{a}$ Kazuhiro Nouso ${ }^{a}$ Akiko Wakuta $^{a}$ Ayano Oonishia \\ Hidenori Toyodab ${ }^{b}$ Toshifumi Tada $^{b}$ Atsushi Hiraoka ${ }^{c}$ Kunihiko Tsuji ${ }^{d}$ \\ Ei Itobayashi ${ }^{\text {e }}$ Toru Ishikawa $^{f}$ Koichi Takaguchig Akemi Tsutsuig \\ Noritomo Shimadah Takashi Kumadab \\ on behalf of the Real-Life Practice Experts for HCC (RELPEC) Study Group \\ and HCC 48 Group (Hepatocellular Carcinoma Experts from 48 Clinics)
}

\begin{abstract}
${ }^{a}$ Department of Gastroenterology and Liver Disease Center, Okayama City Hospital, Okayama, Japan; ${ }^{b}$ Department of Gastroenterology and Hepatology, Ogaki Municipal Hospital, Gifu, Japan; ' ${ }^{\mathrm{C}}$ Gastroenterology Center, Ehime Prefectural Central Hospital, Ehime, Japan; ${ }^{\mathrm{d} C e n t e r}$ of Gastroenterology, Teine Keijinkai Hospital, Hokkaido, Japan; ' Department of Gastroenterology, Asahi General Hospital, Chiba, Japan; ${ }^{f}$ Department of Gastroenterology, Saiseikai Niigata Daini Hospital, Niigata, Japan; ${ }^{9}$ Department of Hepatology, Kagawa Prefectural Central Hospital,

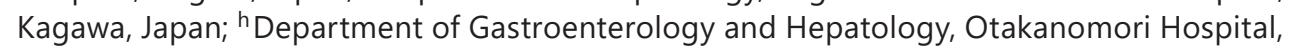
Chiba, Japan
\end{abstract}

\section{Keywords}

Radiofrequency ablation · Surgical resection · Intermediate-stage hepatocellular carcinoma

\begin{abstract}
Background: Transcatheter arterial chemoembolization (TACE) is the standard therapy for intermediate-stage (IM) hepatocellular carcinoma (HCC). However, IM-HCC includes various clinical conditions, and various therapies were conducted in practice. In this study, we retrospectively analyzed the actually conducted treatments for IM-HCC and their efficacies to elucidate the treatment strategies suitable for IM-HCC. Methods: This study included 627 IMHCC of 5,260 HCC from 9 hospitals. We examined the treatment strategies of these patients and analyzed the efficacy of each therapy with the Cox proportional hazard model and propensity score-matched analysis. Results: Liver resection, radiofrequency ablation (RFA), and TACE were performed in 165, 108, and 351 patients, respectively. Liver resection and RFA were preferably selected in cases of Barcelona Clinic Liver Cancer (BCLC)-B1/B2, and patient survival was significantly longer than in those treated with TACE $(p<0.0001)$. However, no beneficial effect of these active therapies was observed in cases of BCLC-B3/B4. Multivariate


analysis revealed that surgical resection (hazard ratio $=0.384$ ) and RFA (hazard ratio $=0.597$ ) were negative risk factors for survival. Propensity score-matching analysis revealed that survival of RFA-treated patients was longer than that of TACE-treated patients ( $p=0.036)$. Conclusion: RFA and surgical resection were effective for IM-HCC, particularly in BCLC-B1/B2 cases.

(C) 2019 S. Karger AG, Basel

\section{Introduction}

Hepatocellular carcinoma (HCC) is the second leading cause of cancer death worldwide and responsible for 788,000 deaths in 2015 [1]. Various treatment modalities and drugs, such as liver resection [2], radiofrequency ablation (RFA) [3-8], transcatheter arterial chemoembolization (TACE) $[9,10]$, tyrosine kinase inhibitors [11-14], and immuno-oncology drugs [15], are used for the treatment of HCC. Several guidelines are proposed to select the treatment modality based on the condition of HCC [16-18]. The Japan Society of Hepatology (JSH) advocated their own JSH guideline in 2017 [18], and the Barcelona Clinic Liver Cancer (BCLC) groups proposed the BCLC guideline in 2018 [16]. Intermediate-stage (IM) hepatocellular carcinoma (HCC) contains various clinical conditions therein and represents a highly heterogeneous population; active discussion is currently ongoing regarding its treatment strategy [17-28]. Based on the aforementioned guidelines, TACE/liver resection/hepatic arterial chemoembolization is recommended for IM-HCC treatment, but local ablation, including RFA, is not recommended $[16,18]$. RFA has good prognosis for HCC up to $3 \mathrm{~cm}$ and 3 nodules, and plays an important role in the current HCC clinical practice [4, 6-8]. In real practice, RFA was often applied to treat IM-HCC, and several reports supported the efficacy of RFA for these patients [23, 26-28]; we have also reported its safety and efficacy in a limited number of patients $[23,28]$. In this study, we conducted a multicenter study to determine the present status of the treatment of IM-HCC in Japan and analyzed the usefulness of curative therapies, including RFA.

\section{Patients and Methods}

\section{Patients}

Subjects enrolled in this analysis were 677 IM-HCC of 5,260 newly discovered HCC cases registered in our collaborative hospitals from 2004 to 2017. The diagnostic criteria for HCC were based on previous reports of hyperattenuation at the arterial phase or hypoattenuation at the portal phase determined using dynamic computed tomography (CT) or magnetic resonance imaging (MRI) [28]. In a few cases with atypical findings, the diagnosis was pathologically confirmed by using tissues obtained by fine-needle tumor biopsy. The sixth-edition tumor, node, metastasis staging for HCC was determined based on previous studies conducted by the Liver Cancer Study Group of Japan (LCSGJ) [30].

We divided the patients into 4 groups: the liver resection, RFA, TACE, and the other therapy group, which included best supportive care. We defined the treatment considered to give the most therapeutic effect as "main treatment." TACE included both conventional TACE and drug-eluting bead TACE.

The study protocol conformed to the ethical guidelines of the World Medical Association Declaration of Helsinki and was approved by our institutional review board (30-152, Okayama City Hospital Institutional Review Board).

\section{Treatments}

Liver resection consists of systematic hepatectomy, partial hepatectomy, and laparoscopic liver resection. RFA was performed solely (69 cases, $63.9 \%$ ) or in conjunction with TACE. We performed RFA in patients in whom TACE was thought to be difficult in treating all nodules or in those who hoped to undergo RFA strongly. When we considered that RFA could be performed safely to all HCC nodules, we explained to the patients that TACE is a standard therapy for IM-HCC and that RFA or RFA combined with TACE might be 
Kariyama et al.: Curative Treatment for Intermediate-Stage HCC

Table 1. Characteristics of the patients with intermediate-stage hepatocellular carcinoma

\begin{tabular}{|c|c|c|c|c|c|c|}
\hline Variables & $\begin{array}{l}\text { Liver } \\
\text { resection }\end{array}$ & RFA & TACE & Others & $p$ value & Total \\
\hline Patients & 165 & 108 & 351 & 53 & & 677 \\
\hline Median age, years & 70 & 69 & 72 & 69 & 0.076 & 71 \\
\hline Gender (male) & $131(79.4 \%)$ & $77(71.3 \%)$ & $276(78.67 \%)$ & $37(69.8 \%)$ & 0.201 & $521(77.0 \%)$ \\
\hline $\mathrm{HBsAg+}$ & $33(20.0 \%)$ & $9(8.3 \%)$ & $34(9.7 \%)$ & $9(17.0 \%)$ & 0.004 & $85(12.6 \%)$ \\
\hline $\mathrm{HCVAb}+$ & $78(47.3 \%)$ & $72(66.7 \%)$ & $214(61.0 \%)$ & 27 (50.9\%) & 0.004 & $391(57.8 \%)$ \\
\hline Child-Pugh A & $155(93.9 \%)$ & $76(70.4 \%)$ & $244(69.5 \%)$ & $20(37.7 \%)$ & $<0.0001$ & $495(73.1 \%)$ \\
\hline \multicolumn{7}{|l|}{ Tumor } \\
\hline Number: $\leq 3 / 4-5 / \geq 6$ & $136 / 17 / 12$ & $29 / 56 / 23$ & $102 / 115 / 134$ & $17 / 16 / 20$ & $<0.0001$ & $284 / 204 / 189$ \\
\hline Size: $<3 / 3-5 />5 \mathrm{~cm}$ & $1 / 77 / 87$ & $41 / 54 / 13$ & $71 / 147 / 133$ & $8 / 18 / 27$ & $<0.0001$ & $121 / 296 / 260$ \\
\hline BCLC stage, B1/B2/B3 & $67 / 92 / 6$ & $42 / 45 / 21$ & $64 / 201 / 86$ & $5 / 17 / 31$ & $<0.0001$ & $178 / 355 / 144$ \\
\hline
\end{tabular}

$n(\%)$ are shown unless indicated otherwise. BCLC, Barcelona Clinic Liver Cancer substage; HBsAg+, positive for hepatitis B virus surface antigen; HCVAb+, positive for hepatitis $\mathrm{C}$ virus antibody.

better than TACE alone in obtaining high local curability, but this effect has not been completely proven yet. All patients in the RFA group agreed to undergo RFA after the explanation.

RFA was performed under ultrasonographic guidance using a single electrode (Cool-tip; Covidien, Tokyo, Japan; Rita-RFA System; Balmer Medical SA, Concise, Switzerland; RF 3000 Radiofrequency Ablation System; Boston Scientific, Marlborough, MA, USA; VIVA-RF system; STARmed, Gyoyang-Si, Korea). TACE was performed on segmental or subsegmental hepatic arteries. A mixture of iodized oil (Lipiodol; Guerbet, Aulnay-sous-Bois, France) and epirubicin hydrochloride (Farmorubicin; Pfizer, Tokyo, Japan), doxorubicin hydrochloride (Adriacin; Pfizer, Tokyo, Japan), or cisplatin (IA-call; Nihon Kayaku, Tokyo, Japan) was initially injected into the arteries, followed by Gelfoam powder (Gelpart; Nihon Kayaku, Tokyo, Japan) or Spongel (before 2006; Yamanouchi, Tokyo Japan).

Follow-Up

We examined the patients every 3-4 months by using simultaneous dynamic CT or Gd-EOB-DTPAenhanced magnetic resonance imaging and assessed tumor markers, such as $\alpha$-fetoprotein (AFP) and protein induced by vitamin K absence or antagonist II (PIVKA-II). The criteria for recurrence were the same as the criteria for initial diagnosis. The mean observation period was 3.2 years.

\section{Statistical Analysis}

Data were expressed as medians and ranges. Statistical analysis was performed using the Mann-Whitney $\mathrm{U}$ test or Wilcoxon test for continuous variables, $\chi^{2}$ test for categorical variables, log-rank test, Kaplan-Meier method, and Cox proportional hazard model for survival. For propensity score-matched analysis, we extracted the data on the patients who underwent TACE or RFA in BCLC-B, matched the background of both groups with age, sex, Child-Pugh score, main tumor size, and tumor number. Statistical significance was considered for $p<0.05$. All statistical analyses were performed using Easy R (EZR; Saitama Medical Center, Jichi Medical University, Saitama, Japan) [16], a graphical user interface for $R$ (The R Foundation for Statistical Computing, Vienna, Austria) [31].

\section{Results}

\section{Treatment Selection}

TACE accounted for the largest number of treatment types in IM-HCC (351 cases, 51.8\%), whereas more than one-third of the patients (273 cases, 39.1\%) underwent curative therapies, such as liver resection or RFA (Table 1; online suppl. Table 1a-c; for all online suppl. material, see www.karger.com/doi/10.1159/000502479). Although liver resection was 


\section{Liver Cancer}

Fig. 1. Treatment selection based on BCLC-B substages. In BCLC-B1, the most prevalent therapy was liver resection (LR; 67 patients, $46.9 \%)$, followed by RFA (42 patients, 29.4\%), and TACE (32 patients, $22.4 \%$ ). The number of TACE increased in BCLC-B2 (84 patients, $40.2 \%$ ); however, more than half of the patients underwent LR or RFA (121 patients, 57.9\%). In BCLC-B3/B4, TACE was preferably selected (86 patients, 59.7\%).

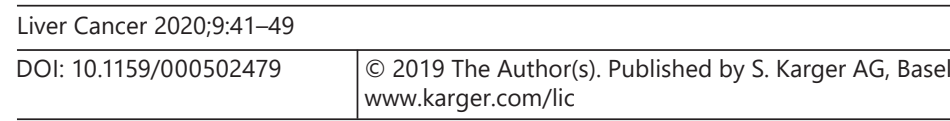

Kariyama et al.: Curative Treatment for Intermediate-Stage HCC

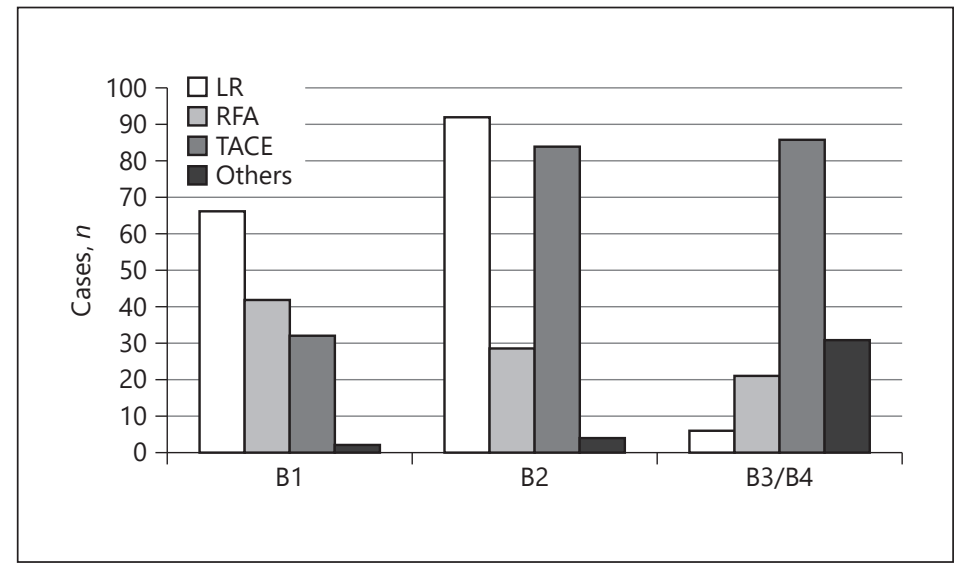

preferably selected for the treatment of large tumors in small number, RFA was used for the treatment of small tumors in large number. No background differences were observed between the groups treated with RFA alone and RFA combined with TACE.

The number of patients in BCLC-B1, -B2, and -B3/4 was 178, 355, and 144, respectively. The most prevalent therapy in BCLC-B1 was liver resection ( 67 patients, $46.9 \%$ ), followed by RFA (42 patients, 29.4\%), and TACE (32 patients, 22.4\%) (Fig. 1). The number of patients treated with TACE increased in BCLC-B2 (84 patients, 40.2\%); however, more than half of the patients underwent liver resection or RFA (121 patients, 57.9\%). TACE was preferably selected in BCLC-B3/B4.

\section{Overall Survival}

The median survival time (MST) by treatment type (liver resection/RFA/TACE/others) in BCLC-B cases was 5.6, 4.2, 2.5, and 1.0 years, and the 5-year survival rate was 55.8, 45.9, 21.1 , and $14.7 \%$, respectively. We analyzed the overall survival of the patients based on the substage of Bolondi et al. [32]. The overall survival of liver resection (MST, 6.43 years) and RFA (MST, 6.96 years) in BCLC-B1 was significantly better than that of TACE (MST, 3.16 years, $p<0.0001$; Fig. 2). The same relationship was observed in BCLC-B2: the overall survival of liver resection (MST, 5.28 years) and RFA (MST, 3.83 years) was significantly better than that of TACE (MST, 2.76 years, $p<0.0001$ ). However, no significant difference in overall survival was observed in BCLC-B3/B4. We did not observe a difference in the prognosis between the RFA-only group (MST 4.19 years) and RFA-TACE group (MST 4.05 years, $p=0.872$ ).

\section{Risk Factors for Survival in Patients with IM-HCC}

Univariate analysis with 15 variables, including tumor factors and background liver factors, revealed that old age ( $>70$ years), low albumin level $(<3.5 \mathrm{~g} / \mathrm{dL})$, high total bilirubin level $(>1.0 \mathrm{mg} / \mathrm{dL})$, presence of ascites and encephalopathy, high AFP level $(>20 \mathrm{ng} / \mathrm{mL})$, high des- $\gamma$-carboxy prothrombin (DCP; $>100 \mathrm{mAU} / \mathrm{mL}$ ), high BCLC substage, and large tumor size were risk factors for survival (Table 2). The cutoff values of AFP and DCP were set based on their median values. Multivariate analysis using the significant factors in the univariate analysis revealed that old age $(>70$ years, hazard ratio $[\mathrm{HR}]=1.324)$, low albumin $(<3.5 \mathrm{~g} /$ $\mathrm{dL}, \mathrm{HR}=1.787)$, and presence of ascites $(\mathrm{HR}=1.390)$ were significant risk factors for survival. By contrast, liver resection $(\mathrm{HR}=0.384)$ and $\mathrm{RFA}(\mathrm{HR}=0.597)$ were negative risk factors for survival. 


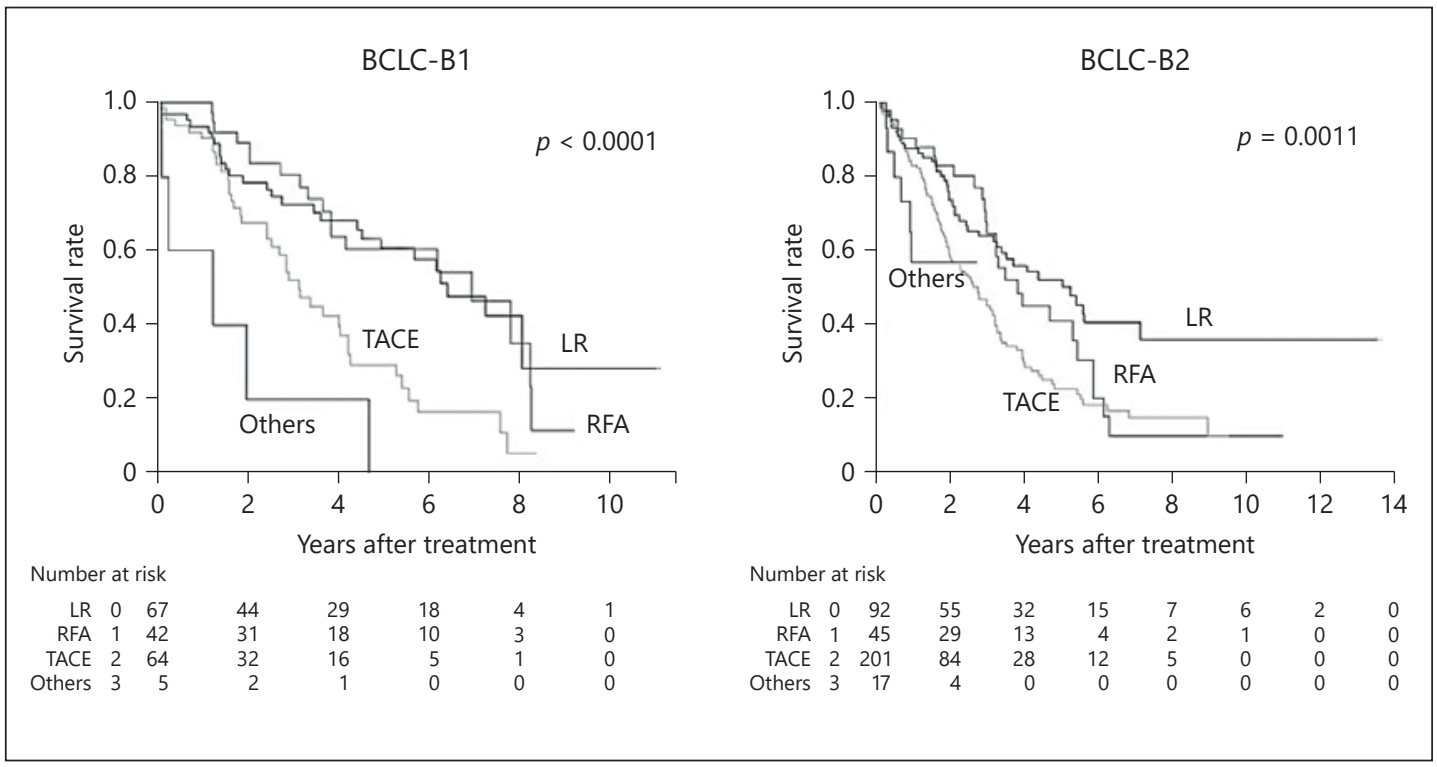

Fig. 2. Overall survival of the patients stratified by BCLC-B substages. In BCLC-B1, overall survival of liver resection (LR; median survival time [MST], 6.43 years) and RFA (MST, 6.96 years) was significantly better than that of TACE (MST, 3.16 years; $p<0.0001$ ). The same relationship was observed in BCLC-B2: MST of LR, RFA, and TACE were 5.28, 3.83, and 2.76 years, respectively $(p<0.0001)$. No significant difference in survival was observed in BCLC-B3/B4 (data not shown).

Table 2. Risk factors for survival

\begin{tabular}{|c|c|c|c|c|c|c|}
\hline \multirow[t]{2}{*}{ Variables } & \multicolumn{3}{|c|}{ Univariate analysis } & \multicolumn{3}{|c|}{ Multivariate analysis } \\
\hline & HR & $95 \%$ CI & $p$ value & HR & $95 \% \mathrm{CI}$ & $p$ value \\
\hline Age $>70$ years & 1.272 & $1.012-1.599$ & 0.039 & 1.324 & $1.044-1.679$ & 0.021 \\
\hline Gender (male) & 1.093 & $0.851-1.403$ & 0.487 & & & \\
\hline HBsAg+ & 1.016 & $0.756-1.365$ & 0.916 & & & \\
\hline $\mathrm{HCVAb}+$ & 0.971 & $0.792-1.190$ & 0.777 & & & \\
\hline Platelets & 0.993 & $0.974-1.012$ & 0.470 & & & \\
\hline Albumin $<3.5 \mathrm{~g} / \mathrm{dL}$ & 1.965 & $1.599-2.415$ & $<0.001$ & 1.787 & $1.400-2.282$ & $<0.001$ \\
\hline T.Bil >1.0 mg/dL & 1.426 & $1.148-1.772$ & 0.001 & & & \\
\hline Ascites present & 1.471 & $1.127-1.919$ & 0.004 & 1.390 & $1.043-1.854$ & 0.025 \\
\hline Encephalopathy present & 1.473 & $1.025-2.116$ & 0.036 & & & \\
\hline $\mathrm{AFP}>20 \mathrm{ng} / \mathrm{mL}$ & 1.413 & $1.112-1.796$ & 0.005 & & & \\
\hline $\mathrm{DCP}>100 \mathrm{mAU} / \mathrm{mL}$ & 1.172 & $1.313-2.232$ & $<0.001$ & & & \\
\hline \multicolumn{7}{|l|}{ Tumor } \\
\hline Size $>30 \mathrm{~mm}$ & 1.438 & $1.105-1.870$ & 0.007 & & & \\
\hline Number $>4$ & 1.169 & $0.953-1.434$ & 0.134 & & & \\
\hline \multicolumn{7}{|l|}{ Treatments } \\
\hline Liver resection & 0.473 & $0.365-0.613$ & $<0.001$ & 0.384 & $0.276-0.536$ & $<0.001$ \\
\hline RFA & 0.660 & $0.498-0.876$ & 0.004 & 0.597 & $0.406-0.847$ & 0.004 \\
\hline
\end{tabular}

AFP, $\alpha$-fetoprotein; AFP-L3, Lens culinaris agglutinin-reactive fraction of AFP; BCLC, Barcelona Clinic Liver Cancer substage; CI, confidence interval; DCP, des- $\gamma$-carboxy prothrombin; HBsAg+, positive for hepatitis B virus surface antigen; HCVAb+, positive for hepatitis C virus antibody; HR, hazard ratio; RFA, radiofrequency ablation; TACE, transcatheter arterial chemoembolization; T.Bil, total bilirubin. 


\section{Liver Cancer}

Fig. 3. Overall survival (after propensity score-matching analysis). The overall survival of patients in the RFA group was significantly longer than that in the TACE group $(p=0.036)$.

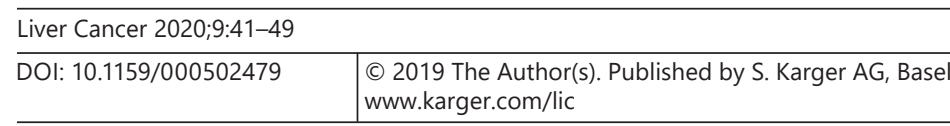

Kariyama et al.: Curative Treatment for Intermediate-Stage HCC

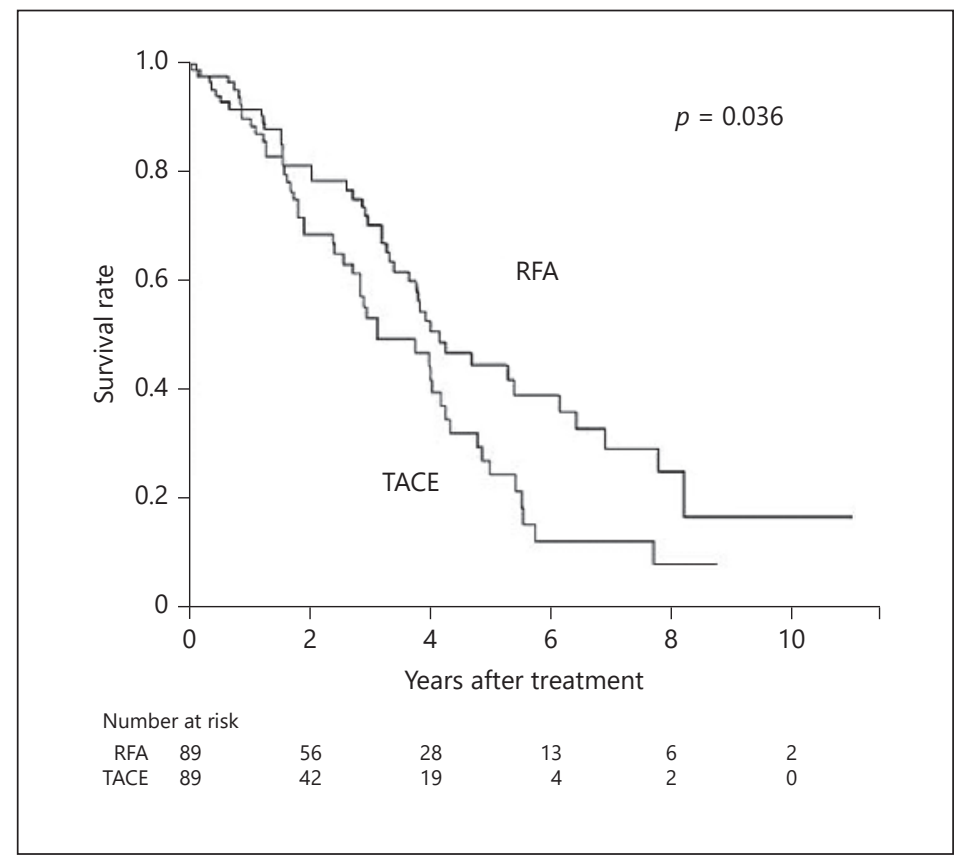

\section{Propensity Score-Matching Analysis of TACE and RFA}

The characteristics of the patients treated with TACE and RFA overlapped to some extent; hence, we matched the background of the patients by using propensity score and compared it with the survival. We used 7 factors for matching (age, sex, Child-Pugh score, tumor number, tumor size, AFP, and DCP), and 89 cases each were selected from 351 TACE and 108 RFA. The c-indices of propensity scores before and after matching were 0.72 and 0.48 , respectively. The average scores in the TACE group and the RFA group were 0.342 (median 0.343, range 0.047-0.632) and 0.356 (median 0.338, range 0.044-0.645), respectively. The overall survival of the patients in the RFA group was significantly longer than that in the TACE group $(p=0.036)$. The MST of the patients in the RFA and TACE groups was 4.19 and 3.17 years, respectively (Fig. 3). The difference was observed especially in BCLC-B1 (online suppl. Fig. 1a, b).

On the other hand, we could not match the background of liver resection and TACE groups completely with propensity score because of the great background differences between them.

\section{Discussion}

In this study, we examined the actual condition of the treatment of IM-HCC by using the data from multiple hospitals in Japan. Approximately $40 \%$ of the patients were treated curatively either with liver resection or RFA, although these treatments were neither recommended in the JSH nor in the BCLC guidelines. These therapies were preferably selected in cases of BCLC-B1/B2 and could achieve longer survival than TACE. However, these active therapies have no beneficial effect in cases of BCLC-B3/B4. Propensity score-matching analysis revealed that survival of the RFA group was longer than that of the TACE group. In addition, liver resection and RFA were negative risk factors for survival in multivariate analysis. These results indicated that RFA and liver resection could be the treatments of choice for IM-HCC, although the candidates must be carefully selected. 


\section{Liver Cancer}

Kariyama et al.: Curative Treatment for Intermediate-Stage HCC

Both liver resection and RFA were often selected in BCLC-B1 and -B2; however, tumor size and number in each group were quite different. The size of the tumor was large in the selected liver resection group, and the number of nodules was high in the RFA group. The beneficial effect of these curative therapies could be achieved only using the advantage of each therapy. A small difference in size did not affect the procedures and curability of liver resection in many cases. A recent study indicated the survival benefit of liver resection in patients with IM-HCC, which included many large $\mathrm{HCC}>5 \mathrm{~cm}$ in diameter with a small number [33]. A small increase in the number of tumors did not dramatically increase the risk or reduce the curability of RFA. Yin et al. [34] showed that RFA could control a large number of tumors but not large tumors. These results indicated that longer survival could be achieved by selecting good candidates for each therapy.

Several studies indicated the beneficial effect of RFA in patients with IM-HCC. Azuma et al. [35] treated HCC with poor Lipiodol retention with RFA, and Yin et al. [34] performed RFA to nodules that might be safe to perform RFA. Both studies showed the additive effect of RFA for prolonging survival to patients initially treated with TACE. However, most of the studies did consider the BCLC-B substages. We previously reported in a different small cohort that the treatment with RFA alone was better than TACE alone in BCLC-B1/B2 for the first time [23]. Hirooka et al. demonstrated the additive effect of RFA in BCLC-B1/2 patients who received TACE [26]. The results in this multicenter study supported the conclusions of both studies.

Although there are good candidates for RFA in patients with BCLC-B1/B2, surgical resection and RFA has no beneficial effect in cases of BCLC-B3/4. These patients were not only bearing more advanced tumors but also experiencing worse liver function. The efficacy of any treatments, including TACE, has not been confirmed in patients with poor liver function; hence, research trials were required to propose suitable therapies [32].

This study has some limitations. First, this study is not a randomized controlled but a retrospective cohort study. Selection bias could not be eliminated because liver resection and RFA were performed only when these therapies were considered safe and beneficial. In addition, we neither considered recently developed tyrosine kinase inhibitors nor PD-1 antibodies. These drugs were originally used for the treatment of advanced HCC (BCLC-C); however, its expanded application to IM-HCC is now on the stage, particularly for TACErefractory cases. These drugs might change the strategy for the treatments of IM-HCC in the near future. We did not examine the effect of posttreatments, which might be another limitation. Furthermore, we did not take tumor location into account, which might be a cause of selection bias.

Despite these limitations, we clearly demonstrated the benefit of liver resection and RFA for the treatment of BCLC-B1/B2 IM-HCCs in this multicenter analysis. The results in this study could provide information for selecting therapies of IM-HCCs.

\section{Acknowledgments}

The authors gratefully acknowledge all the doctors who collaborated on this project by collecting data of the patients with HCC in their affiliated hospitals.

\section{Statement of Ethics}

The study protocol conformed to the ethical guidelines of the World Medical Association Declaration of Helsinki and was approved by our institutional review board (30-152; Okayama City Hospital Institutional Review Board). 


\section{Disclosure Statement}

The authors have no conflicts of interest to declare.

\section{Funding Sources}

This research received no external funding.

\section{Author Contributions}

Conceptualization, T.K.; data curation, H.T., T.T., A.H., K.T., E.I., T.I., A.T., N.S., A.W., A.O. and T.K.; methodology, T.K.; writing - original draft, K.K.; writing - review and editing, K.N.

\section{References}

1 Global Burden of Disease Cancer Collaboration, Fitzmaurice C, Allen C, Barber RM, Barregard L, Bhutta ZA, Brenner H, Dicker DJ, et al. Global, regional, and national cancer incidence, mortality, years of life lost, years lived with disability, and disability-adjusted life-years for 32 cancer groups, 1990 to 2015: a systematic analysis for the Global Burden of Disease Study. JAMA Oncol. 2017 Apr;3(4):524-48.

2 Keen WW. IV. Report of a Case of Resection of the Liver for the Removal of a Neoplasm, with a table of seventysix cases of resection of the liver for hepatic tumors. Ann Surg. 1899 Sep;30(3):267-83.

3 Shiina S, Teratani T, Obi S, Hamamura K, Koike Y, Omata M. Nonsurgical treatment of hepatocellular carcinoma: from percutaneous ethanol injection therapy and percutaneous microwave coagulation therapy to radiofrequency ablation. Oncology. 2002;62(1 Suppl 1):64-8.

4 Tateishi R, Shiina S, Teratani T, Obi S, Sato S, Koike Y, et al. Percutaneous radiofrequency ablation for hepatocellular carcinoma. An analysis of 1000 cases. Cancer. 2005 Mar;103(6):1201-9.

5 Shiina S, Teratani T, Obi S, Sato S, Tateishi R, Fujishima T, et al. A randomized controlled trial of radiofrequency ablation with ethanol injection for small hepatocellular carcinoma. Gastroenterology. 2005 Jul;129(1):12230.

6 Shiina S, Tateishi R, Arano T, Uchino K, Enooku K, Nakagawa H, et al. Radiofrequency ablation for hepatocellular carcinoma: 10-year outcome and prognostic factors. Am J Gastroenterol. 2012 Apr;107(4):569-77; quiz 578.

7 Kudo M. Local ablation therapy for hepatocellular carcinoma: current status and future perspectives. J Gastroenterol. 2004;39(3):205-14.

8 Kudo M. Radiofrequency ablation for hepatocellular carcinoma: updated review in 2010. Oncology. 2010 Jul; 78 Suppl 1:113-24.

9 Yamada R, Nakatsuka H, Nakamura K, Sato M, Itami M, Kobayashi N, et al. [Transcatheter arterial embolization therapy for hepatic tumor (author's transl)]. Rinsho Hoshasen. 1981 Jan;26(1):41-8.

10 Yamada R, Sato M, Nakatsuka H, Nakamura K, Shibakiri I, Itami M, et al. [Transcatheter hepatic artery embolization in 60 patients with hepatocellular carcinoma studies on angiographic features (author's transl)]. Nihon Igaku Hoshasen Gakkai Zasshi. 1981;41(8):742-50.

11 Llovet JM, Ricci S, Mazzaferro V, Hilgard P, Gane E, Blanc JF, et al.; SHARP Investigators Study Group. Sorafenib in advanced hepatocellular carcinoma. N Engl J Med. 2008 Jul;359(4):378-90.

12 Kudo M, Finn RS, Qin S, Han KH, Ikeda K, Piscaglia F, et al. Lenvatinib versus sorafenib in first-line treatment of patients with unresectable hepatocellular carcinoma: a randomised phase 3 non-inferiority trial. Lancet. 2018 Mar;391(10126):1163-73.

13 Kudo M. Extremely high objective response rate of lenvatinib: its clinical relevance and changing the treatment paradigm in hepatocellular carcinoma. Liver Cancer. 2018 Sep;7(3):215-24.

14 Hiraoka A, Kumada T, Kariyama K, Takaguchi K, Itobayashi E, Shimada N, et al.; Real-life Practice Experts for HCC (RELPEC) Study Group and the HCC 48 Group (hepatocellular carcinoma experts from 48 clinics in Japan). Therapeutic potential of lenvatinib for unresectable hepatocellular carcinoma in clinical practice: multicenter analysis. Hepatol Res. 2019 Jan;49(1):111-7.

15 Kudo M. Immuno-Oncology in Hepatocellular Carcinoma: 2017 Update. Oncology. 2017;93 Suppl 1:147-59.

16 Galle PR, Forner A, Llovet JM, Mazzaferro V, Piscaglia F, Raoul JL, et al; European Association for the Study of the Liver. Electronic address: easloffice@easloffice.eu; European Association for the Study of the Liver. EASL Clinical Practice Guidelines: Management of hepatocellular carcinoma. J Hepatol. 2018 Jul;69(1):182-236.

17 Omata M, Cheng AL, Kokudo N, Kudo M, Lee JM, Jia J, et al. Asia-Pacific clinical practice guidelines on the management of hepatocellular carcinoma: a 2017 update. Hepatol Int. 2017 Jul;11(4):317-70. 
Kariyama et al.: Curative Treatment for Intermediate-Stage HCC

18 Kudo M. Management of Hepatocellular Carcinoma in Japan as a World-Leading Model. Liver Cancer. 2018 May; $7(2): 134-47$.

19 Labgaa I, Demartines N, Melloul E. Surgical Resection Versus Transarterial Chemoembolization for Intermediate Stage Hepatocellular Carcinoma (BCLC-B): An unsolved question. Hepatology. 20182019 Feb;69(2): 923.

20 Matsukuma S, Sakamoto K, Tokuhisa Y, Tokumitsu Y, Matsui H, Kanekiyo S, et al. Outcomes following liver resection for multinodular Barcelona Clinic Liver Cancer-B hepatocellular carcinoma. Oncol Lett. 2018 Nov; 16(5):6383-92.

21 Kamo N, Kaido T, Yagi S, Okajima H, Uemoto S. Liver Transplantation for Intermediate-Stage Hepatocellular Carcinoma. Liver Cancer. 2018 May;7(2):179-89.

22 Tada T, Kumada T, Toyoda H, Tsuji K, Hiraoka A, Itobayashi E, et al. Role of hepatic resection in patients with intermediate-stage hepatocellular carcinoma: A multicenter study from Japan. Cancer Sci. 2017 Jul;108(7): 1414-20.

23 Nouso K, Kariyama K, Nakamura S, Oonishi A, Wakuta A, Oyama A, et al. Application of radiofrequency ablation for the treatment of intermediate-stage hepatocellular carcinoma. J Gastroenterol Hepatol. 2017 Mar;32(3): 695-700.

24 Pecorelli A, Lenzi B, Gramenzi A, Garuti F, Farinati F, Giannini EG, et al.; Italian LiverCancer (ITA.LI.CA) group. Curative therapies are superior to standard of care (transarterial chemoembolization) for intermediate stage hepatocellular carcinoma. Liver Int. 2017 Mar;37(3):423-33.

25 Ogasawara S, Chiba T, Ooka Y, Kanogawa N, Motoyama T, Suzuki E, et al. Efficacy of sorafenib in intermediatestage hepatocellular carcinoma patients refractory to transarterial chemoembolization. Oncology. 2014; 87(6):330-41.

26 Hirooka M, Hiraoka A, Ochi H, Kisaka Y, Joko K, Michitaka K, et al. Transcatheter arterial chemoembolization with or without radiofrequency ablation: outcomes in patients with barcelona clinic liver cancer stage b hepatocellular carcinoma. AJR Am J Roentgenol. 2018 Apr;210(4):891-8.

27 Tanaka M, Ando E, Simose S, Hori M, Kuraoka K, Ohno M, et al. Radiofrequency ablation combined with transarterial chemoembolization for intermediate hepatocellular carcinoma. Hepatol Res. 2014 Feb;44(2):194200.

28 Kariyama K, Wakuta A, Nishimura M, Kishida M, Oonishi A, Ohyama A, et al. Percutaneous Radiofrequency Ablation for Intermediate-Stage Hepatocellular Carcinoma. Oncology. 2015;89 Suppl 2:19-26.

29 Kudo M, Kitano M, Sakurai T, Nishida N. General rules for the clinical and pathological study of primary liver cancer, nationwide follow-up survey and clinical practice guidelines: the outstanding achievements of the Liver Cancer Study Group of Japan. Dig Dis. 2015 Oct;33(6):765-70.

30 Liver Cancer Study Group of Japan. The General Rules for the Clinical and Pathological Study of Primary Liver Cancer. 6th ed. Tokyo: Kanehara \& Co; 2015. pp. 26-30.

31 R Development Core Team RFfSC. R: A Language and Environment for Statistical Computing. Vienna: The R Foundation for Statistical Computing; 2005.

32 Bolondi L, Burroughs A, Dufour JF, Galle PR, Mazzaferro V, Piscaglia F, et al. Heterogeneity of patients with intermediate (BCLC B) Hepatocellular Carcinoma: proposal for a subclassification to facilitate treatment decisions. Semin Liver Dis. 2012 Nov;32(4):348-59.

33 Hyun MH, Lee YS, Kim JH, Lee CU, Jung YK, Seo YS, et al. Hepatic resection compared to chemoembolization in intermediate- to advanced-stage hepatocellular carcinoma: A meta-analysis of high-quality studies. Hepatology. 2018 Sep;68(3):977-93.

34 Yin X, Zhang L, Wang YH, Zhang BH, Gan YH, Ge NL, et al. Transcatheter arterial chemoembolization combined with radiofrequency ablation delays tumor progression and prolongs overall survival in patients with intermediate (BCLC B) hepatocellular carcinoma. BMC Cancer. 2014 Nov;14(1):849.

35 Azuma S, Asahina Y, Nishimura-Sakurai Y, Kakinuma S, Kaneko S, Nagata H, et al. Efficacy of additional radiofrequency ablation after transcatheter arterial chemoembolization for intermediate hepatocellular carcinoma. Hepatol Res. 2016 Apr;46(4):312-9. 